\title{
Aproximación a la función de los asentamientos precerámicos en la Puna y su borde oriental (Jujuy, Argentina)
}

Hugo D. YACOBACCIO ${ }^{1}$

\section{Introducción}

El presente trabajo propone evaluar algunos aspectos de los asentamientos de cazadores recolectores ubicados en la Puna oriental y su borde (prePuna) en la Provincia de Jujuy.

Nosotros pensamos que un estudio funcional del asentamiento puede brindar datos para sustanciar modelos económicos y de movilidad al estimarse con certidumbre elementos que representan a las actividades desarrolladas en dichos lugares.

Se ha explicitado que los contextos representan las actividades desarrolladas en los sitios donde se hallan y la variabilidad de los mismos representaría, en parte, las diferentes tareas que en ellos se llevaron a cabo (Binford 1973). Un argumento principal aquí es que ciertos conjuntos de artefactos (toolkits) representan ciertas actividades generales. El aislamiento de dichos conjuntos ha sido una preocupación del "argumento funcional" desde que fue planteado (Binford y Binford 1966). Whallon (1973) ha sugerido medios estadísticos para distinguir conjuntos que covarían espacialmente. Discutiremos algunos de ellos más adelante.

La información manejada por nosotros proviene de cinco sitios estratificados emplazados en los tramos superiores de las quebradas de acceso del valle de Humahuaca hacia la Puna, y uno de ellos sobre una quebrada secundaria a la de Yacoraite a los pies orientales de la serranía Aguilar. Se han obtenido siete fechados radiocarbónicos que se agrupan en dos "momentos": uno hacia ca. 9200 AP y otro hacia 3500 AP. Sin duda, esto no representa una distribución real y de ninguna manera debe interpretarse como una falta de habitación del lugar entre los extremos cronológicos mencionados. Esta

1 Instituto de Ciencias Antropológicas, Facultad de Filosofía y Letras, Universidad de Buenos Aires, 25 de Mayo $2171^{\circ}$, 1002 Buenos Aires, ARGENTINA. distribución se debe, en la actualidad, a la falta de un registro más completo.

La base de este estudio será el desarrollo del análisis funcional de los artefactos líticos y espacial del sitio Inca Cueva 4. Del resto poseemos datos parciales extraídos de los informes de excavación y, por lo tanto, la metodología se adecuará a tales circunstancias. De Inca Cueva 7 hemos tenido acceso a los materiales y a su estudio más detallado. En los siguientes acápites cada sitio se tratará por separado.

\section{Inca Cueva 4}

El sitio está ubicado en el tramo superior de la quebrada homónima a $3800 \mathrm{~m} . \mathrm{snm}$ que se abre en las areniscas rojas del subgrupo Pirgua (Cretácico), a $70 \mathrm{~m}$ del margen derecho del arroyo y a $15 \mathrm{~m}$ del nivel de base local. Está orientado hacia el este, y tiene $17.60 \mathrm{~m}$ de frente y $6.50 \mathrm{~m}$ de profundidad máxima.

La capa de ocupación 2 es la que nos interesa. Está caracterizada por un sedimento limoso con abundante paja desmenuzada y camadas de paja compactada (Stipa sp., Bromus sp. y Festuca sp.) en la base del estrato. Los residuos carbonosos le dan una coloración gris. Se asienta sobre una capa de arenas rojizas sin vestigios orgánicos. La secuencia de formación de la capa 2 es la siguiente:

1) Excavación de un piso habitacional en el piso estéril de la capa 3 (arenas rojizas) por los ocupantes de la cueva.

2) Ubicación de haces de paja compactados a modo de piso y depositación de artefactos y residuos orgánicos.

La estructura de la planta ya ha sido descrita preliminarmente (Aschero 1979). Los habitantes de la cueva formatizaron una hoyada extrayendo una capa de 12 a $14 \mathrm{~cm}$ de espesor en un diámetro de $3 \mathrm{~m}$. Dentro de esta hoyada se excavaron dos "pozones" contiguos (Y y Z) (Figura 1); "Y" mide 85 x $62 \mathrm{~cm}$ 
por $39 \mathrm{~cm}$ de profundidad y "Z" 43 x $66 \mathrm{~cm}$ por $38 \mathrm{~cm}$ de profundidad. En el interior de los mismos no había paja compactada sino desmenuzada y gran cantidad de elementos "suntuarios" (cuentas de columela, colgantes de pezuña de cérvido, plumas de colores embarriladas y la mayor parte de los fragmentos de cestería). En la periferia de la hoyada se encontraban pequeños hoyos con un diámetro regular $(20 \mathrm{~cm})$ y una profundidad variable entre 11 y $23 \mathrm{~cm}$. Ubicados diagonalmente en el borde interno y externo de los pozones, se encontraban dos pequeños fogones planos (Figura 1b). Las camadas de paja tenían un espesor de $1-2 \mathrm{~cm}$ y se adelgazaban progresivamente hacia los bordes de la estructura. ${ }^{2}$

Para el estudio funcional del sitio hemos empleado una metodología doble: en primer lugar, se analizó microscópicamente cada artefacto recuperado para detectar huellas de uso; en segundo lugar, se realizó un estudio de la distribución espacial de los ítems en la planta. Esto fue facilitado dado que fueron mapeados in situ la totalidad de elementos recuperados. Deben descontarse de este mapeo los artefactos hallados en el sondeo preliminar del año 1978 pero que igualmente pueden ser referidos a una localización de $0.75 \mathrm{~m}^{2}$.

El estudio de microdesgaste fue referido en trabajos anteriores (Yacobaccio 1979 y 1982); por lo tanto, haremos aquí un resumen de los resultados obtenidos. Un rasgo sobresaliente del sitio es la excelente conservación del material orgánico. Esto permitió que en casi todos los instrumentos se detectaran sustancias adheridas a los filos que revelaban de manera clara la actividad en la cual habían sido empleados. La mayoría de los artefactos presentan pelos de camélidos adheridos a sus filos; otros muestran una sustancia grasa cenicienta. El pelo está adherido manteniendo una misma estructura en todos los casos presentando: 1) una capa de grasa entre la roca y el pelo, 2) pelo brilloso, y 3 ) ausencia de partículas de cuero entre el pelo. De acuerdo a trabajos experimentales en raspado de pieles secas, mojadas y untadas con grasa, las características

2 En 1982 se amplió la excavación hacia los bordes laterales de la hoyada, encontrándose una extensión de la capa 2 y nuevas estructuras (dos pozones laterales) conteniendo estos restos orgánicos, principalmente vegetales. Sin embargo, la densidad de artefactos disminuye en forma sensible. Hasta ahora puede plantearse que la hoyada funcionó como una unidad y las tareas desarrolladas en su periferia no la afectaron mayormente, aunque aquellas pueden ser receptáculo de limpiezas periódicas de material descartado en la hoyada. mencionadas se repiten en esta última. Asimismo, la evidencia etnográfica analizada incluye esta práctica (untar con grasa u otra sustancia grasosa el pelo al ser trabajado) en forma recurrente. Esta evidencia nos llevó a plantear que dichos instrumentos fueron destinados al trabajo de piel untada con grasa y secada con cenizas (capa cenicienta sobre algunos artefactos), con el fin de confeccionar elementos de cuero curtido sin pelos tales como bolsas, "tientos", etc. Esta afirmación se ve fortalecida por el hecho de hallarse en el piso de habitación fragmentos de cuero curtido y sin curtir y gran cantidad de vellones sueltos, que según evidencia experimental se conforman como residuos del raspado. Asimismo se recuperó gran cantidad de vellones de pelo de Cervidae, pero éstos no se hallan adheridos a los filos de los instrumentos. Por lo tanto, planteamos una técnica de tratamiento diferente a la empleada para el camélido que no incluye untar la misma con grasa (Yacobaccio 1982).

De igual manera, una menor cantidad de artefactos reveló signos de desgaste (abrasión y pulimentos) característicos del trabajo sobre vegetales no leñosos. Esta tarea puede asociarse al trabajo de conformación del piso de Gramineae.

Sintetizando, entonces, la actividad principal realizada en Inca Cueva 4 fue el trabajo sobre pieles de camélido y cérvido y que de acuerdo a la evidencia ósea disponible hasta el momento (ausencia casi total de huesos de estos animales) debían ser llevadas en procesamiento al sitio. El resto de las tareas está correlacionado con la actividad principal (confección de bolsas, tientos, etc.) y con la subsistencia normal basada principalmente en Chinchillidae (Mengoni 1982) y recursos vegetales locales de recolección, fundamentalmente soldaque (Hysocharis tridentata), una raíz tuberosa comestible.

Así dada la referencia funcional de los artefactos, el segundo paso consiste en ubicarlas espacialmente. Hemos mencionado que se han sugerido medios estadísticos para distinguir conjuntos de artefactos que covarían espacialmente. Según Cahen y colaboradores (1979: 662) este método se basa en los siguientes presupuestos: 1) que los instrumentos que covarían positivamente funcionan juntos; 2) que los instrumentos que covarían negativamente no funcionan juntos; 3) que las tipologías reflejan funciones, y 4) que la tecnología "expeditiva" predomina sobre la "conservada". Estos autores consideran no investigadas convenientemente dichas proposiciones, si bien el argumento general es correcto, hay dificultades en su aplicación particular. 


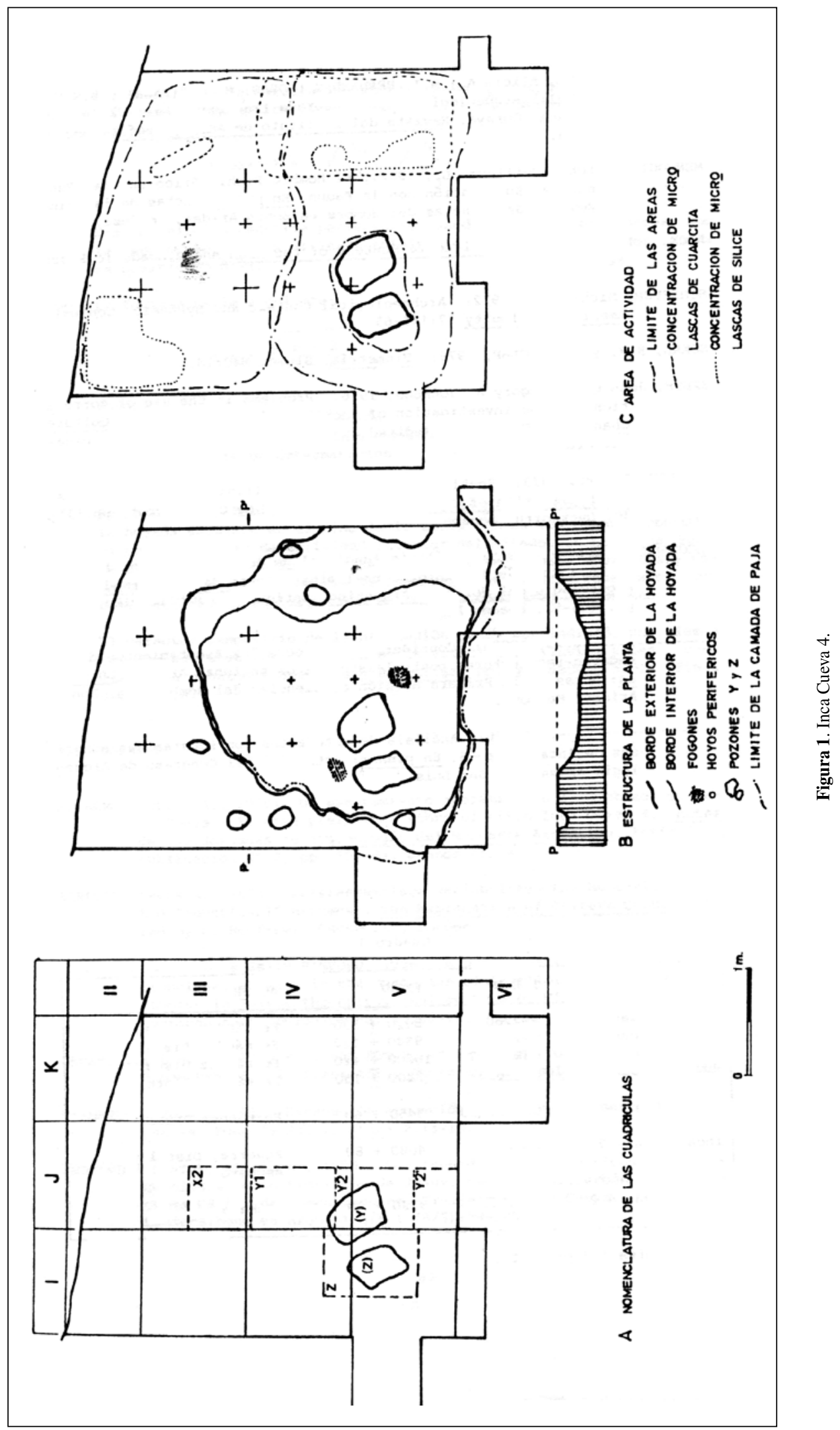


El problema más importante, pensamos, es la determinación de conjuntos de artefactos con significado funcional a partir de clasificaciones tipológicas morfológicas. El estudio de microdesgaste por uso permite, al contrario, la adscripción funcional de los conjuntos posibilitando la elaboración de cadenas funcionales. De todos modos, el empleo de ciertos coeficientes se hace necesario con el fin de verificar la asociación espacial de los elementos y evitar el efecto de la asociación por azar.

Múltiples factores inciden en la formación del contexto arqueológico y, por supuesto, en su asociación espacial. Muchos de estos factores no están directamente vinculados a las actividades que se desarrollaron en el sitio, sino también a perturbaciones postdepositacionales (Schiffer 1972). En todos los casos se busca diferenciar los factores intervinientes y determinar asociaciones espaciales no azarosas con significado funcional definidas como áreas de actividad.

En Inca Cueva 4 no hemos detectado perturbaciones postdepositacionales drásticas; por lo tanto, la distribución espacial observada corresponde aproximadamente al estado que presentaba el sitio al ser abandonado por sus ocupantes. El coeficiente empleado para distinguir áreas de actividad ha sido el de correlación. Con él se trata de medir el grado de asociación entre dos variables. Hemos empleado el coeficiente de producto-momento, también conocido como "r de Pearson" (Sokal y Rolhf 1979: 543). ${ }^{3}$ Sin embargo, la formación de pares para el análisis presenta problemas. Hay que tener en cuenta que existen factores que pueden influenciar el signo y la magnitud del coeficiente y que afectan su habilidad para distinguir patrones espaciales.

3 La fórmula de coeficiente "r de Pearson" es la siguiente:

$$
r=\frac{\sum \frac{x-\bar{x}}{S_{x}} \frac{y-\bar{y}}{S_{y}}}{n}
$$

donde:

$x$ e $y \quad$ : son los valores medidos para las dos variables.

$\bar{x}$ e $\bar{y}:$ son los valores medios de su distribución.

$S_{x}$ y $S_{y}$ : las desviaciones típicas correspondientes.

El resultado está comprendido entre $-1 \mathrm{y}+1$. Estos valores extremos indican, en el primer caso, una correlación negativa (no hay asociación entre las variables) y +1 representa una correlación positiva perfecta. Cero indica una correlación nula.
Si se incrementa la variabilidad en la frecuencia de los ítems por unidad, aunque se mantenga una segregación espacial total de estos, la magnitud de la correlación negativa decrece abruptamente. La presencia de pares 0-0 (ausencia de ambas clases) puede conducir a este error e incluso al cambio de signo. Esta situación se presenta por varios motivos: 1) cuando ciertas clases de artefactos son raras; 2) alrededor del perímetro del sitio donde decrece la cantidad de material, y 3) en zonas de baja densidad entre loci de depositación (Speth y Johnson 1976).

Atendiendo a esto, en la confección de los pares de variables hemos eliminado las celdas $0-0$ y no consideramos en los casos que presenten dichas celdas, a los sectores (no llegan a conformar una cuadrícula de 1 x $1 \mathrm{~m}$ ) perimetrales: numerados II y VI (interno y externo respectivamente) (Figura 1a). Nuestra unidad espacial de referencia para componer los pares fue el microsector de $0.50 \times 0.50 \mathrm{~m}$, medida compatible con los $0.75 \times 0.75 \mathrm{~m}$ de las cuadrículas excavadas en 1978 y que, como dijimos, no teníamos ubicación exacta de los elementos en planta.

Si las distribuciones de frecuencias de dos clases de artefactos son diferentes o asimétricas el rango máximo posible del coeficiente de correlación se contraerá y será mucho menor que \pm 1 . Este rango de contracción se puede ilustrar confeccionando el coeficiente phi $(\phi)$. Este es una forma especial de "r de Pearson" en el cual los datos han sido dicotomizados en presencia-ausencia ó $0-1{ }^{4}$

Para controlar la información así obtenida y establecer los límites de confianza del coeficiente se empleó el test " $t$ " para verificar si un coeficiente

4 La fórmula phi es como sigue:

donde:

$$
\phi \max (+)=+\sqrt{\left(\frac{p_{j}}{q_{j}} \frac{q_{i}}{p_{i}}\right)}
$$

$p_{i}$ : la proporción marginal mayor dada en la tabla 2 × 2

$p_{j} \quad$ : la proporción marginal menor.

Si $p_{x}$ es la proporción marginal mayor se convierte en $p_{i}$ y $p_{y}$ es $p_{j}$ y así de seguido. Si $q_{y}$ es la proporción marginal mayor entonces es $p_{i}$ y $q_{x}$ es $p_{j}$




de correlación muestral puede provenir de una población con un coeficiente paramétrico igual a cero (Sokal y Rolhf 1979: 564). Esto significa que la hipótesis nula es:

$$
\mathrm{H}_{0}: \mathrm{p}=0
$$

lo que implica que las variables no están correlacionadas. Por el contrario, entonces, nuestra hipótesis alternativa fue:

$$
\mathrm{H}_{1}: \mathrm{p} \neq 0
$$

lo que implica que las variables están correlacionadas. ${ }^{5}$

Siguiendo a Speth y Johnson (1976) se pueden postular los siguientes patrones espaciales (Cuadro 1):

1. Areas de actividad específica. Los artefactos están divididos en distintas unidades o loci, correspondiendo cada uno a una actividad simple o a un grupo de actividades relacionadas. Los artefactos fueron descartados aproximadamente en el lugar donde fueron usados.

2. Areas de actividad no específicas. Se desarrollaron actividades no relacionadas en las mismas unidades o loci.

3. Areas de depósito aglomerada. Items que representan un gran rango de actividades, pueden ser depositados y subsecuentemente abandonados.

4. Areas de depositación aglomeradas. Pueden resultar del vaciamiento o barrido de instrumentos descartados y otros desechos en un área relativamente compacta adyacente a loci residenciales, o a lo largo del perímetro del sitio, o de un área de actividad principal.

Así, la magnitud y signo esperado de la correlación de acuerdo a estas definiciones son los siguientes:

\begin{tabular}{|c|l|l|}
\hline $\begin{array}{c}\text { Distribuciones } \\
\text { espaciales }\end{array}$ & \multicolumn{1}{|c|}{$\begin{array}{c}\text { Instrumentos son } \\
\text { funcionalmente específicos }\end{array}$} & $\begin{array}{c}\text { Instrumentos son } \\
\text { funcionalmente no específicos }\end{array}$ \\
\hline Tipo 1 & $\begin{array}{l}\text { (+) débil a fuerte } \\
(-) \text { débil }\end{array}$ & $\begin{array}{l}\text { (+) débil a fuerte } \\
(-) \text { no esperado }\end{array}$ \\
\hline Tipo 2 & $\begin{array}{l}(+) \text { fuerte } \\
(-) \text { no esperado }\end{array}$ & $\begin{array}{l}\text { (+) débil a fuerte } \\
(-) \text { no esperado }\end{array}$ \\
\hline $\begin{array}{l}\text { Tipo 3 } \\
\text { a. por residencia }\end{array}$ & $\begin{array}{l}(+) \text { débil a fuerte } \\
(-) \text { no esperado } \\
\text { b. por función }\end{array}$ & $\begin{array}{l}(+) \text { débil a fuerte } \\
(-) \text { no esperado }\end{array}$ \\
\hline Tipo 4 & $\begin{array}{l}\text { (+) débil a fuerte } \\
(-) \text { débil o no esperado }\end{array}$ & $\begin{array}{l}\text { (+) débil a fuerte } \\
(-) \text { no esperado }\end{array}$ \\
\hline
\end{tabular}

Cuadro 1. Patrones espaciales.

donde:

$p_{x}$ es la proporción de grillas que contienen $x$

$q_{x}$ es la proporción de grillas que no contienen $x$.

$\mathrm{p}_{x y}$ es la proporción de grillas que contienen $x$ e $y$ etc.

El coeficiente de correlación es +1 cuando $p_{x}=p_{y} \neq q_{x}=q_{y}$. Ecuación similar se aplica para averiguar el rango máximo de coeficientes negativos:

$$
\phi \max (-)=-\sqrt{\left(\frac{q_{i}}{p i}\right.} \quad \sqrt{\left.\frac{q_{j}}{p_{j}}\right)}
$$

Una correlación negativa perfecta es cuando $p_{x}=p_{y}$. Si tenemos que las proporciones de $x$ difieren totalmente de las de $y$ entonces nunca es posible +1 ó -1 .

5 La fórmula es la siguiente:

$$
t=r \sqrt{\frac{n-2}{1-r^{2}}}
$$

De acuerdo a estos presupuestos, entonces, hemos elaborado correlaciones de: instrumentos de corte instrumentos de raspado; instrumentos - microlascas, para todo el sitio y para el área denominada fogones/ pozones y "reservada". ${ }^{6}$ Igualmente, para microlascas de cuarcita - microlascas de sílice e instrumentos "suntuarios". Los resultados obtenidos se muestran en el Cuadro 2:

$$
\begin{aligned}
& \text { donde: } \\
& \mathrm{r} \quad=\text { coeficiente de correlación. } \\
& \mathrm{n}-2=\text { grados de libertad. } \\
& \mathrm{n} \quad=\text { número de pares. } \\
& \mathrm{r}^{2} \quad=\text { coeficiente de determinación }
\end{aligned}
$$

6 Aschero (1979) había reconocido un área de habitación conformada por la hoyada excavada en el piso de la cueva. A su vez estaría constituida por un espacio de actividad interior delimitado por el locus donde se había recuperado la mayor cantidad de artefactos y ecofactos y caracterizado por la presencia de fogones, y un espacio reservado con baja densidad de hallazgos que se infirió como área de descanso. 


\begin{tabular}{|c|c|c|c|c|}
\hline Pares* & $\mathbf{r}$ & $\phi(p h i)$ & $\mathbf{t}$ & p $\left(\right.$ rechazo de $\left.H_{0}\right)$ \\
\hline A & .296 & $\max (+): .910$ & - & - \\
\hline $\mathrm{B}$ & .379 & $\max (+): .804$ & .709 & No rechaza $\mathrm{H}_{0}$ \\
\hline $\mathrm{C}$ & .574 & $\max (+): .829$ & 1.21 & No rechaza $\mathrm{H}_{0}$ \\
\hline $\mathrm{D}$ & .573 & - & 3.27 & $\mathrm{p}<005$ \\
\hline $\mathrm{E}$ & .686 & - & 4.31 & $\mathrm{p}<001$ \\
\hline $\mathrm{F}$ & -.231 & $\max (-): .209$ & .487 & No rechaza $\mathrm{H}_{0}$ \\
\hline G & -.125 & - & .218 & No rechaza $\mathrm{H}_{0}$ \\
\hline
\end{tabular}

Cuadro 2. Correlaciones *A: corte-raspado; B: corte-raspado área fogones/pozones; C: corte-raspado área "reservada"; D: raspadomicrolascas; E: corte-microlascas; F: microlascas sílice-microlascas cuarcita; G: instrumentos (general)-"suntuarios" área fogones/pozones.

La débil asociación registrada entre instrumentos de corte y raspado indica especificidad areal para realizar estas tareas. Esto se ve confirmado en la medida que el test "t" es incapaz de rechazar la hipótesis nula. Esta apreciación se fundamenta, asimismo, en el no rechazo de la misma en el área "reservada" y en que los instrumentos recuperados en ese locus son funcionalmente específicos destinados casi todos a raspado de piel, dado que sólo tres fueron utilizados en trabajo sobre vegetal no leñoso y dos a perforado. De aquí se puede deducir que en el lugar se llevaron a cabo tareas relacionadas con la conformación de pieles especialmente despelado.

En el caso de los pares de instrumentos de corte y microlascas e instrumentos de raspado y microlascas, la hipótesis nula es rechazada. Esto indica covariación. A su vez, el no rechazo de la $\mathrm{H}_{0}$ en relación a las microlascas de sílice y cuarcita confirma el resultado negativo de la correlación, planteando una distribución diferencial para ambas. Esto apuntaría a sostener, asimismo, un origen diferente. Mientras unas serían subproducto de actividades de talla independiente (sílice; presencia de instrumentos no terminados), las otras, lo serían del trabajo sobre pieles (la materia prima de los artefactos destinados a esta tarea son de cuarcita) (Figura 1c: línea de puntos).

En el área de fogones/pozones no se verifica la asociación entre instrumentos (en conjunto) y artefactos "suntuarios" (par G). El coeficiente de determinación, es decir, que los segundos dependan de los primeros, es muy bajo $\left(r^{2}=.0144\right)$. Esta situación puede indicar que dichos artefactos no fueron confeccionados en el sitio, sino sólo depositados en él. A su vez, el test "t" no rechaza la hipótesis nula avalando lo dicho. Refuerza este razonamiento la localización exclusiva de los mismos (152 ítems en los pozones y sólo tres fuera de ellos).
Estas consideraciones permiten formular la existencia de un área de actividad específica comprendida más o menos por la llamada "área reservada" destinada al trabajo de piel como tarea principal y, en menor medida, a actividades sobre vegetal no leñoso y poca talla lítica. Un área de depositación aglomerada conformada por el área de fogones/pozones y originada, tal vez, por el descarte de artefactos provenientes del locus anterior y un área de depósito conformada por los pozones Y y Z (Figura 1c).

\section{Funciones estimadas para los otros sitios del área}

Intentaremos ahora ofrecer una interpretación funcional del resto de los sitios de la zona. Un examen de los contextos hallados puede verse en Yacobaccio (1982a), por lo tanto resumiremos la información a las variables pertinentes. Las dataciones radiocarbónicas obtenidas pueden observarse en el Cuadro 3.

\section{Huachichocana (CH III)}

Dos niveles precerámicos han sido detectados en este sitio (E3 y E2), separados por una distancia temporal apreciable. La ocupación más antigua presenta elementos que hacen pensar en el desarrollo de actividades de talla, principalmente la confección de puntas de proyectil triangulares y lanceoladas pequeñas, que constituyen el elemento mayormente representado (Fernández Distel 1974). Actividades secundarias pudieron ser el procesamiento de fibras vegetales y cueros. La manufactura in situ de la cordelería y cestería presente es otra posibilidad aunque más remota dado el carácter de descarte de estos artefactos. Otra alternativa es agregada por la inhumación secundaria con ofrendas pertenecientes a este nivel, aunque puede pertenecer a otro evento de ocupación. La ocupación de la subcapa 


\begin{tabular}{|l|l|l|l|}
\hline Sitio / Nivel & Lab. $\mathbf{N}^{\mathbf{0}}$ & Años AP & \multicolumn{1}{c|}{ Autor } \\
\hline Huachichocana E3 & P-2280 & $8420 \pm 530$ & Fernández Distel \\
\hline Huachichocana E3 & P-2236 & $9340 \pm 120$ & Fernández Distel \\
\hline Huachichocana E3 & Gak-5847 & $10200 \pm 420$ & Fernández Distel \\
\hline Huachichocana E2 & Gak-6357 & $3400 \pm 130$ & Fernández Distel \\
\hline Peña Aujero D & GrN-10014 Distel \\
\hline Inca Cueva 7 & T-1773 & $3450 \pm 60$ & Aguerre y colaboradores \\
\hline Inca Cueva 4 2 & CSIC-498 & $4080 \pm 80$ & Aschero \\
\hline
\end{tabular}

Cuadro 3. Dataciones obtenidas para los sitios del área.

E2 está caracterizada por sus “conjuntos fúnebres" (Fernández Distel 1980: 95). Según la autora citada representa una continuación tecnoeconómica de la ocupación anterior aunque con indicaciones de mayor movilidad (Fernández Distel 1980: 96). El sitio puede ser caracterizado como un campamento de actividades más generalizadas aunque temporario con enterratorios y ofrendas. ${ }^{7}$

\section{Peña Aujero (capas B, C y D)}

La confección y reemplazo de puntas líticas triangulares parece ser la tarea principal dada la recuperación preponderante de este tipo de artefactos, algunos inconclusos, junto a una cantidad relativamente grande de microlascas. La zona excavable es reducida en relación a la superficie del abrigo (Fernández Distel et al. 1981), por lo tanto, las consideraciones efectuadas son parciales. De todos modos, la escasa densidad de artefactos como de restos óseos muy fracturados podrían indicar una corta ocupación. El sitio pudo ser utilizado como campamento temporario con actividad de talla.

\section{Abrigo I del río Despensas}

Los datos son escasos. Se registraron puntas de proyectil triangulares y raederas en una ocupación de escasa densidad. El único registro de una mano de molino (Fernández 1975) podría indicar actividades de molienda. El sitio puede ser tipificado como un campamento temporario con actividades específicas.

\footnotetext{
7 El carácter temporario de la habitación está indicado por elementos que sugieren una ocupación en la estación de lluvias tales como camélidos nonatos y neonatos y Passiflora sp. que madura hacia fines del verano.
}

\section{Inca Cueva 7}

Se trata de un evento único de depositación de una selección de elementos ergológicos que indican la variedad del total de tareas realizables, tal vez en distintos sitios. La mayoría del material lítico, por ejemplo, está compuesto por puntas de proyectil lanceoladas pequeñas $(n=24)$, algunas de las cuales fueron empleadas y recuperadas y otras permanecieron sin usarse. En el sitio no se llevó a cabo ninguna tarea específica, sólo la depositación de estos elementos, por lo que puede ser caracterizado como un sitio de ofrenda.

Las estimaciones ofrecidas más arriba están basadas sobre datos cualitativos y pueden variar si se realizan análisis más exhaustivos. De todos modos, son indicadores suficientes para especificar líneas generales del uso de los sitios.

\section{Consideraciones generales}

Algunas implicaciones de este análisis pueden plantearse en forma tentativa para ser contrastadas en el futuro. Rick (1980) ha brindado una serie de características a los dos tipos de sitios (campamentos base y secundarios) empleados para sustanciar el modelo de sedentarismo propuesto para la Puna de Junín (Perú).

Nuestra propuesta para la zona que nos ocupa, en cambio, apuntaría a denotar que cierta especificidad funcional de los sitios estaría representando patrones de movilidad que articularían una gama de sitios dedicados a la explotación del ecosistema local y concurrentemente a la realización de trabajos determinados. De aquí se puede esperar que: 
1) En cada sitio estará representada una tarea predominante. El resto de las tareas estará subordinado o será complementario de la primera. No habrá un rango amplio de actividades representadas.

2) La tarea predominante podrá excluir, en ciertos casos, las actividades de subsistencia normales de la explotación del ecosistema local.

3) La fauna y la flora locales estarán más conspicuamente representadas que los elementos alóctonos.

4) La densidad de ocupación deberá ser restringida.

5) La variedad tipológica del instrumental no será acentuada.

6) Sólo partes seleccionadas de animales de gran porte (p.e., Camelidae y Cervidae) estarán presentes.

7) Los sitios multicomponentes podrán presentar variaciones funcionales a lo largo de la secuencia.

8) Deberá haber cambios estacionales relacionados a las actividades de los sitios.
Así podrán obtenerse dos tipos generalizados de sitios: 1) campamentos temporarios de funciones específicas, y 2) campamentos temporarios de funciones generalizadas. En el primer caso, se trata de sitios ocupados esporádicamente o incluso hasta estacionalmente (una estación completa o gran parte de ella). Estos tipos de sitios pueden ser canteras y/o talleres, sitios de acecho de caza $\mathrm{y}$ todos los sitios anteriormente examinados. La segunda categoría incluye sitios ocupados más allá de una sola estación y estarían más cerca de lo que se conoce como campamento-base. Es importante tener en cuenta que si se toma la existencia de un campamento-base, el cumplimiento del modelo de trashumancia obligaría al abandono del mismo por parte del grupo durante un lapso prolongado, por lo menos hasta una estación al año.

En la región de la Puna y su borde oriental (Jujuy) se cuenta, hasta el momento, con una sola categoría general de sitios: campamentos de funciones específicas. Por lo tanto, la contrastación efectiva del modelo sólo podrá realizarse en el futuro, a medida que aumente la variabilidad sitios representados. Este estudio sólo es una vía hacia ese camino.

\section{REFERENCIAS CITADAS}

ASCHERO, C., 1979. Un asentamiento acerámico en la Quebrada de Inca Cueva. Informe preliminar sobre el sitio Inca Cueva 4. Actas de las Jornadas de Arqueología del Noroeste Argentino, Antiquitas 2: 159-83.

BINFORD, L., 1973. Interassemblage variability. The Musterian and the "functional" argument. En The explanation of culture change, C. Renfrew (Ed.). Duckworth, Londres.

BINFORD, L. y S. BINFORD, 1966. A preliminary analysis of functional variability in the Musterian of Levallois facies. En Recent studies in paleoanthropology, J. D. Clark y F. C. Howell (Eds.). American Anthropologist 68 (2): 238-95.

CAHEN, D., L .H. KEELEY y F. L. VAN NOTEN, 1979. Stone tools, tool kits, and human behavior in prehistory. Current Anthropology 20 (4): 661-83.

FERNANDEZ, J., 1975. Excavación arqueológica en los abrigos del río Despensas, Puna de Jujuy. Etnia 22: 3-10.

FERNANDEZ DISTEL, A., 1980. Los fechados radiocarbónicos de la arqueología de la Provincia de Jujuy. Fechas radiocarbónicas de la cueva CH III de Huachichocana, Tiuiyaco e Inca Cueva. Argentina Radiocarbono en Arqueología I (4/5): 89-100.
FERNANDEZ DISTEL, A., M. I. HERNANDEZ LLOSAS, M. CASIRAGHI y B. VENRA, 1981. Arqueología de una quebrada transversal al valle de Humahuaca: Coraya. Revista del Instituto de Antropología 36.

MENGONI, G., 1982. El hombre prehistórico en la Puna argentina: Su relación con la fauna. Actas de la Primera Reunión de Ciencias del Hombre en Zonas Aridas, Mendoza. En prensa.

RICK, J., 1980. Prehistoric hunters of the High Andes. Academic Press, Nueva York.

SCHIFFER, M., 1972. Archaeological context and systemic context. American Antiquity 37: 156-65.

SOKAL, R. y F. ROLHF, 1979. Biometría. Blume, Madrid.

SPETH, J. y G. JOHNSON, 1976. Problems in the use of correlation for the investigation of toolkits and activity areas. En Culture change and continuity, C. Cleland (Ed.), pp. 35-57. Academic Press, Nueva York.

WHALLON, R., 1973. Spatial analysis of paleolithic occupation areas. En The explanation of culture change, C. Renfrew (Ed.), pp. 115-30. Duckworth, Londres. 
YACOBACCIO, H. D., 1979. Análisis funcional de los instrumentos líticos de Inca Cueva 4. Informe preliminar. Actas de las Jornadas de Arqueología del Noroeste Argentino, Antiquitas 2: 146-152.

_ 1982a. Consideraciones sobre los asentamientos de cazadores recolectores post-pleistocénicos en zonas áridas. Actas de la
Primera Reunión de Ciencias del Hombre en Zonas Aridas, Mendoza. En prensa.

- 1982b. Análisis de artefactos con sustancias adheridas de Inca Cueva 4. Actas del VII Congreso de Arqueología Argentina, San Luis. En prensa. 